\section{MS44-P4 Diffuse scattering analysis of sodium fluorosilicate}

Erik Stronks ${ }^{1}$, Hans-Beat Bürgi ${ }^{1,2}$, Anthony Linden ${ }^{1}$

\author{
MS44-P5 Diffuse scattering in the high Tc \\ superconductor, $\mathrm{HgBa}_{2} \mathrm{CuO}_{4+\delta}$. \\ Thomas R. Welberry ${ }^{1}$
}

1. Australian National University, Research School of Chemistry

1. Department of Chemistry, University of Zürich, Winterthurerstrasse 190, CH-8057 Zürich

2. Department of Chemistry and Biochemistry, University of Bern, Freiestrasse 3, CH-3012 Bern

\section{email: erik.stronks@chem.uzh.ch}

Many crystalline materials with interesting properties show correlated disorder, e.g. relaxor ferroelectrics and super ionic conductors ${ }^{1}$. Diffuse X-ray scattering analysis is one of few means to elucidate the crystal structure at the nano- and micrometer length scale. However, methods to derive a disorder model, including both data processing and analysis, are strongly lagging behind those for average structure determination. We will present some data processing techniques and our attempts at modeling the diffuse scattering of crystalline sodium fluorosilicate. The diffuse scattering data of $\mathrm{Na}_{2} \mathrm{SiF}_{6}$ has been symmetry averaged using the Laue group $-3 m 1$. Outlier rejection based on Blessing ${ }^{2}$ has been applied in order to remove artifacts and spurious peaks. For background correction we have developed a least squares procedure by which the background is estimated from the data without the need of background measurements. A user definable set of spherical harmonic functions of degree $l$ and order $m$ are used as the least squares basis functions. The background estimate, $B$, can be expressed as:

$$
B\left(r^{r e c}, \theta, \varphi\right)=\operatorname{Rad}\left(r^{r e c}\right) \sum \sum A_{l m} Y_{l m}(\theta, \varphi)
$$

here $B$ is the background fit which is a function of the distance from the reciprocal space origin $r^{r e c}$, and the two polar angles $\theta$ and $\varphi$. Rad is a radial function describing the radial dependence of the background intensity, $A_{l m}$ are the set of coefficients to be determined in the least squares procedure and $Y_{l m}$ are the real spherical harmonic functions that where provided by the user. The first sum is over the spherical harmonic degrees $l$ from $l=0$ to $l$ and the second sum is over spherical harmonic orders $m$ from $m=-l$ to $l$. Figure $l$ shows the symmetry averaged $h k 0$ layer before and after background correction. In order to be able to use $3 \mathrm{D}-\triangle \mathrm{PDF}^{3}$ analysis for disordered crystal structure modeling the Bragg peaks would ideally have to be deconvoluted from the diffuse intensies. We will present our attempts at doing so.

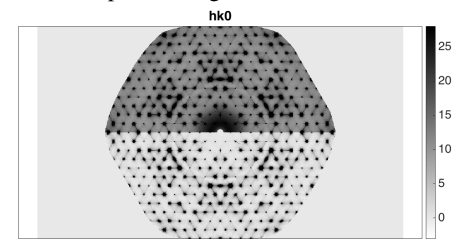

Figure 1. Symmetry averaged hk0 layer before (top) and after (bottom) background correction

Keywords: background fitting, least squares email: welberry@rsc.anu.edu.au

The properties of many technologically important materials are intimately associated with the inherent disorder that exists in their crystal structures. Examples include: alloys, shape-memory alloys, ferroelectrics, fast ion conductors, semi-conductors, to high Tc superconductors, zeolites and inclusion compounds and even pharmaceuticals. To understand these materials it is insufficient to know their average unit cell structure as revealed by Bragg scattering. It requires additional knowledge of their local or nanoscale structure information that can only be obtained from the diffuse scattering component of the total scattering. It is perhaps quite surprising that while there have been many studies of single-crystal diffuse scattering for alloys and ferroelectric materials, for example, there have been very few for high $T \mathrm{c}$ superconductors. However, Izquierdo et al [1] have recently reported quite detailed diffuse scattering patterns obtained from a single crystal of $\mathrm{HgBa}_{2} \mathrm{CuO}_{4+\delta}$, showing that for these materials too such information is quite accessible.

However, though obtaining diffuse scattering data is now feasible for most crystalline materials, interpreting and analyzing the data remains problematical. Izquierdo et al developed a model to try to explain the diffraction patterns they had observed but although their 'best fit' model captured some of the attributes of the observed diffraction pattern, some key features were poorly modeled. In the work described we have developed a model that satisfactorily accounts for the details of the observed diffraction pattern. This is significantly different from the published model and hence has implications for understanding the structure-property relationship of this important material.

[1] Izquierdo et al. (2011), Journal of Physics and Chemistry of Solids, 72: 545-548.

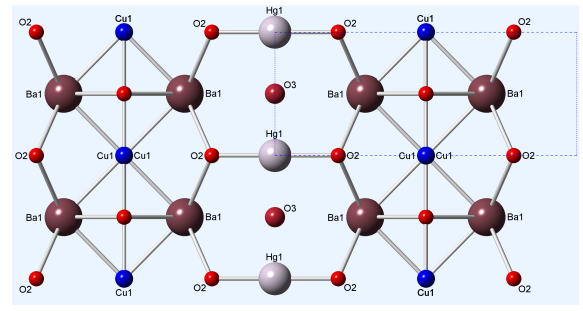

Figure 1. The average structure of the high $T_{C}$ superconductor, $\mathrm{HgBa}_{2} \mathrm{CuO}$. View down [010] showing how the planar $\mathrm{HgO}_{\delta}$ layer is sandwiched between two $\mathrm{Ba}_{2} \mathrm{CuO}_{4}$ layers.

Keywords: Diffuse scattering, SCDS, high temperature superconductivity, computer simulation, bond-valence 